\title{
Highlights from the TESLA Technical Design Proposal
}

\author{
O. Napoly (for the TESLA collaboration) \\ CEA/Saclay, Gif-sur-Yvette, France
}

\begin{abstract}
The technical design report (TDR) of the TESLA superconducting linear collider with an integrated X-ray FEL facility, has been published in the spring of 2001 by an international collaboration. It includes a description of the high energy physics programme, a proposal for the detector, and the collider design relying on the technical results from the TESLA Test Facility (TTF). We review the results achieved by the TESLA collaboration on the superconducting RF technology and beam acceleration at the TTF linac. We highlight some aspects of the collider design and briefly describe the cost study attached to the TDR.
\end{abstract}

\section{OVERVIEW}

Aiming at c.m. energies at the $\mathrm{TeV}$ scale with luminosities around $10^{34} \mathrm{~cm}^{-2} \mathrm{~s}^{-1}$, the next generation of linear colliders require at least 100 times higher beam powers and 100 times smaller beam sizes at the collision point as compared to the SLAC Linear Collider (SLC).

The fundamental characteristic of the TESLA [1] approach is the choice of superconducting accelerating structures. TESLA uses 9-cell Niobium cavities (Fig.1) cooled by superfluid Helium to $T=2 \mathrm{~K}$ and operating at $1.3 \mathrm{GHz}$ $\mathrm{RF}$ frequency. The design gradient at $E_{c m}=500 \mathrm{GeV}$ is $E_{a c c}=23.4 \mathrm{MV} / \mathrm{m}$. Because the power dissipation in the cavity walls is extremely small, the accelerating field can be produced with long, low peak power RF-pulses. This results in a high RF to beam power transfer efficiency, allowing a high average beam power while keeping the electrical power consumption within acceptable limits $(\sim 100 \mathrm{MW})$. Secondly, extremely small beam sizes at the interaction point (IP) require preserving ultra-small emittance beams in the linac. This is well suited with the relatively low frequency (large dimensions) of the TESLA cavities and their accordingly weak wakefields (beam-cavity interaction).

The long bunch train with a large bunch spacing ( $337 \mathrm{ns)}$ permitted by the superconducting RF-pulse $(1 \mathrm{~ms})$ is also beneficial from three respects:

- A fast (MHz) bunch-to-bunch feedback can be used to correct the orbit within one beam pulse. Such a feed-

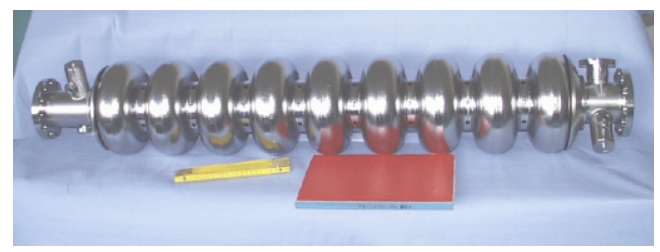

Figure 1: The 9-cell Niobium cavity for TESLA.
Table 1: TESLA parameters for $E_{c m}=500 \mathrm{GeV}$.

\begin{tabular}{lc}
\hline Accelerating gradient, $E_{a c c}[\mathrm{MV} / \mathrm{m}]$ & 23.4 \\
RF-frequency, $f_{R F}[\mathrm{GHz}]$ & 1.3 \\
Fill factor & 0.747 \\
Total site length, $L_{t o t}[\mathrm{~km}]$ & 33 \\
Active length $[\mathrm{km}]$ & 21.8 \\
\# of acc. structures & 21024 \\
\# of klystrons & 584 \\
Klystron peak power $[\mathrm{MW}]$ & 9.5 \\
Repetition rate, $f_{r e p}[\mathrm{~Hz}]$ & 5 \\
Beam pulse length, $T_{P}[\mu \mathrm{s}]$ & 950 \\
RF-pulse length, $T_{R F}[\mu \mathrm{s}]$ & 1370 \\
\# of bunches per pulse, $n_{b}$ & 2820 \\
Bunch spacing, $\Delta t_{b}[\mathrm{~ns}]$ & 337 \\
Charge per bunch, $N_{e}\left[10^{10}\right]$ & 2 \\
Emittance at IP, $\gamma \epsilon_{x, y}\left[10^{-6} \mathrm{~m}\right]$ & $10,0.03$ \\
Beta at IP, $\beta_{x, y}^{*}[\mathrm{~mm}]$ & $15,0.4$ \\
Beam size at IP, $\sigma_{x, y}^{*}[\mathrm{~nm}]$ & 553,5 \\
Bunch length at IP, $\sigma_{z}[\mathrm{~mm}]$ & 0.3 \\
Beamstrahlung, $\delta_{E}[\%]$ & 3.2 \\
Luminosity, $L_{e+e}\left[10^{34} \mathrm{~cm}^{-2} \mathrm{~s}^{-1}\right]$ & 3.4 \\
Power per beam, $P_{b}[\mathrm{MW}]$ & 11.3 \\
Primary electric power, $P_{A C}[\mathrm{MW}]$ & 105 \\
\hline
\end{tabular}

back system will maintain the beams in collision at the IP, making TESLA relatively insensitive to mechanical vibrations which could otherwise lead to serious luminosity reduction.

- In the event of an emergency, a fast safety system can "turn off" the beam within a fraction of a pulse.

- Each bunch collision can be tagged by the detector and the detector background can be isolated.

Making use of these unique features has led to a parameter set (table 1) which clearly demonstrates TESLA's potential for high luminosity.

Upgrading the collider up $800 \mathrm{GeV} \mathrm{cm}$ energy on the same site requires increasing the accelerating gradient up to $35 \mathrm{MV} / \mathrm{m}$, well below the $50 \mathrm{MV} / \mathrm{m}$ fundamental limit for Niobium structures at $2 \mathrm{~K}$, together with a slightly closer packing of the RF cavities as provided by the susperstruture concept (see Sect.2). A higher luminosity can be obtained by raising the beam and RF powers : it therefore requires doubling the number of klystrons and approximately doubling the cooling capacity the cryogenic plants.

\section{STATUS OF R\&D ON SC RF CAVITIES}

In order to demonstrate the feasibility of the highgradient cavity technology and to create a solid basis for a 


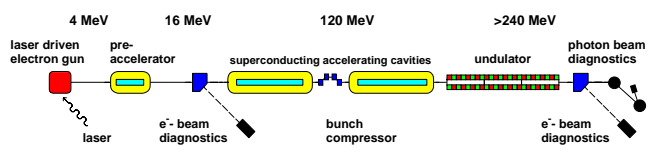

Figure 2: Schematic layout of the TESLA Test Facility Linac (TTFL).
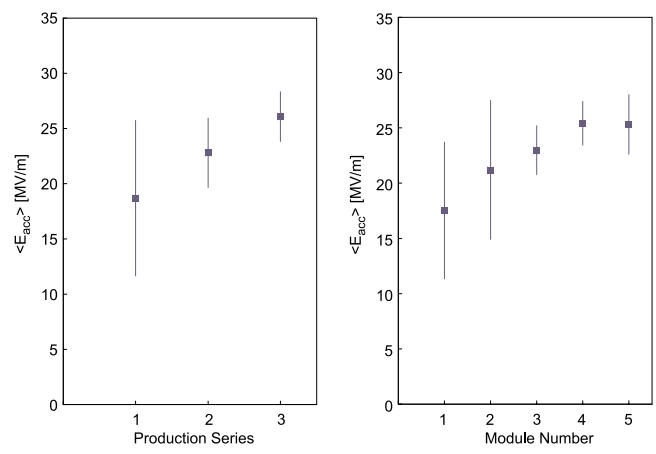

Figure 3: Average accelerating gradients at $Q_{0} \geq 10^{10}$ measured in the vertical test cryostat of: (a) the cavities in the three production series; and (b) the cavities installed in the first five cryogenic modules for TTF.

reliable cost estimate of future large-scale production, the TESLA collaboration is running an $R \& D$ program centered around the TESLA Test Facility (TTF) [2]. The TTF includes the infrastructure for applying different processing techniques to the Niobium cavities obtained from industrial production and a $120 \mathrm{~m}$ long linac (Fig.2) designed, built and commissioned by the members of the TESLA collaboration.

As shown by Figs.3,4, gradients in excess of $25 \mathrm{MV} / \mathrm{m}$ are regularly obtained in large series of industrially produced TESLA cavities. In the third cryomodule installed in the TTF linac, the maximum usable gradient was determined for each of the eight cavities : the average gradient was $23.6 \mathrm{MV} / \mathrm{m}$, indicating that installation of the individual cavities into a module does not degrade their performance. A typical TESLA $800 \mu$ s long $8 \mathrm{~mA}$ beam pulse has been accelerated in the TTF linac at moderate gradient: with a $10 \%$ charge variation along the pulse the $\mathrm{RF}$ control and beam loading compensation systems achieved a relative energy stability of $0.07 \%$ RMS [3].

The energy upgrade to $800 \mathrm{GeV}$ uses of the so-called 'superstructure', where the effective acceleration length is increased by combining two multicell cavities (see Fig.5) with a reduced inter-cavity spacing $\lambda_{R F} / 2$ and, the number of RF-input couplers is halved by powering them with a single high power coupler. The accelerating field gradient required is then $35 \mathrm{MV} / \mathrm{m}$ which, although already approached by a few exceptional TTF cavities, represents a significant increase compared to the performance achieved so far. Such high gradients have been reached in a reproducible manner in single-cell cavities, as shown by Fig.6, by applying a new cavity preparation procedure investi-

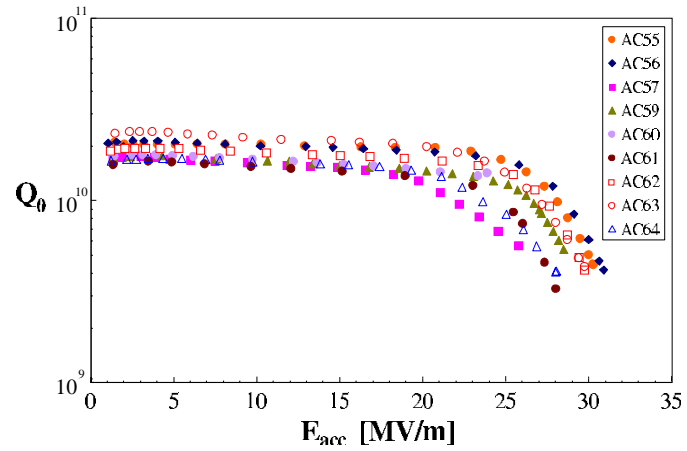

Figure 4: Excitation curve of cavities of the $3^{\text {rd }}$ production series.

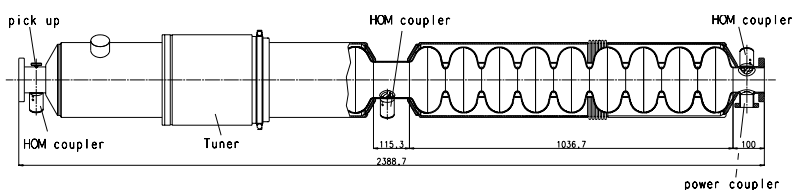

Figure 5: Superstructure II consisting of two 9-cell resonators. The power coupler is at one end.

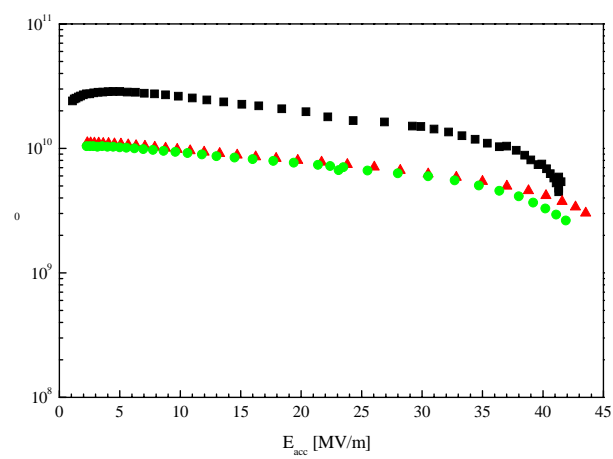

Figure 6: Excitation curves of three electropolished singlecell cavities with baking at $120^{\circ} \mathrm{C}$ baking.

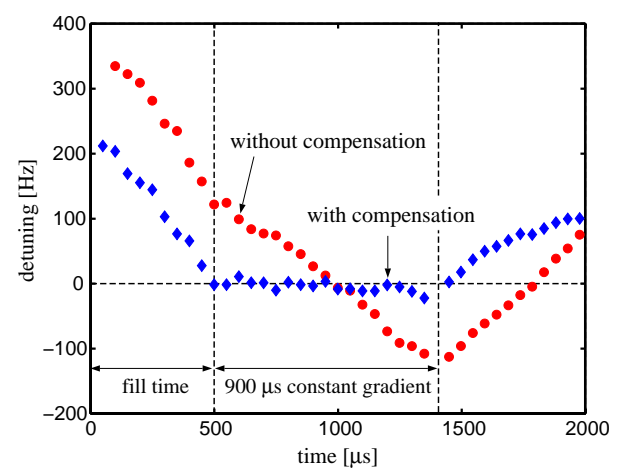

Figure 7: Piezoelectric compensation of the Lorentz-force induced frequency shift $\left(E_{\mathrm{acc}}=23.5 \mathrm{MV} / \mathrm{m}\right.$. 


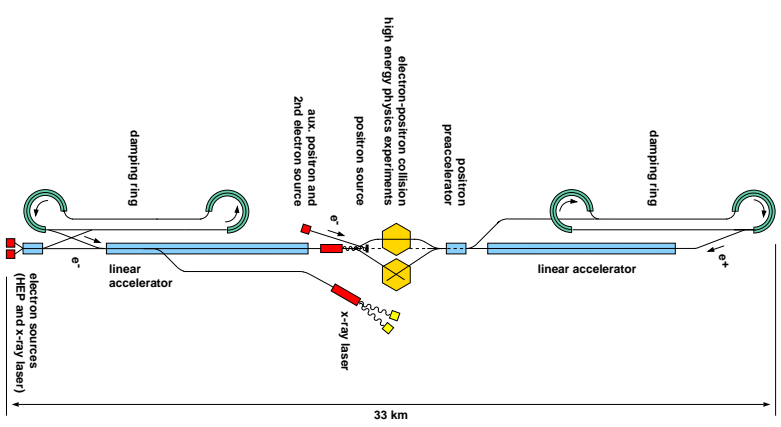

Figure 8: Sketch of the overall layout of TESLA.

gated jointly by KEK, CERN, DESY and Saclay. It involves 'electropolishing' by which the RF surface material is removed in an acid mixture under current flow, followed by clean water rinsing and baking at moderate temperature $\left(100-150^{\circ} \mathrm{C}\right)$. This new method might allow to omit the $1400^{\circ} \mathrm{C}$ heat treatment which prevails in the current production.

The RF detuning of the cavity induced by its mechanical deformation due to the internal electromagnetic radiation pressure, has been identified as an outstanding problem beyond $25 \mathrm{MV} / \mathrm{m}$ gradient. Below this gradient the current mechanical stiffening of the multicell cavity is sufficient. To allow for higher gradients the stiffening must be improved, or alternatively, the cavity deformation must be compensated. The latter approach has been successfully demonstrated using a piezoelectric tuner (see Fig.7). The result indicates that the present stiffening augmented by a piezoelectric tuning system will permit efficient cavity operation at the TESLA- 800 gradient of $35 \mathrm{MV} / \mathrm{m}$.

\section{COLLIDER DESIGN}

The TESLA collider layout is shown in Fig.8. It includes an X-ray free electron laser user facility which exploits the first $50 \mathrm{GeV}$ section of the electron linac and requires two extraction and bypass lines parallel to the main linac to allow an energy variability, and hence FEL wavelength tuneability, from 13 to $50 \mathrm{GeV}$. We discuss here only the $e^{+} e^{-}$ collider main sub-systems.

\subsection{Injection System and Damping Rings}

The electron beam is generated in a polarized laserdriven gun based on the GaAs photocathode technology developed for the SLC[4] where the electron polarization reached $80 \%$.

The positron injection system has to provide a total charge of about $5.6 \cdot 10^{13} e^{+}$per beam pulse, which is not feasible with a conventional (electron on thick target) source. Instead, positrons are produced from $\gamma$-conversion in a thin target (see Fig.9). The photons are generated by passing the high-energy electron beam through an undulator placed after the main linac, before transporting the beam to the IP. Passage through the undulator causes the energy

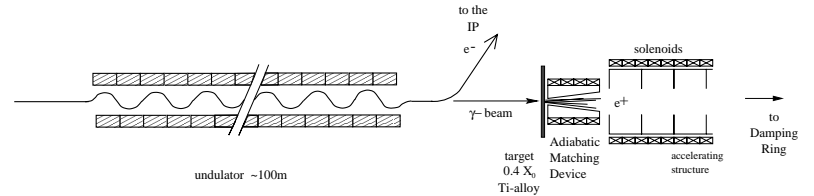

Figure 9: Sketch of the positron source layout.

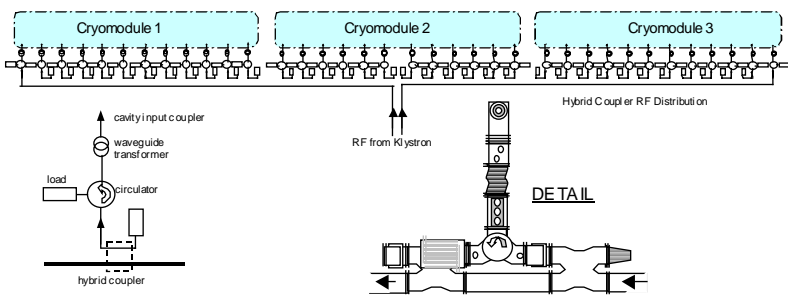

Figure 10: RF waveguide distribution of one RF station

spread in the electron beam to increase from $0.5 \cdot 10^{-3}$ to $1.5 \cdot 10^{-3}$, with an average energy loss of $1.2 \%$, both of which appear tolerable. Besides providing a sufficiently high positron beam intensity, the undulator-based source offers several advantages: a) use of a thin target leads to a smaller positron beam with a smaller transverse emittance than from a conventional (thick target) source; b) polarized positrons is possible by replacing the planar undulator with a helical undulator. The polarised positron option is technically more ambitious and is considered a potential upgrade at a later stage of operation. The achievable polarisation ranges from $45 \%$ to $60 \%$. The undulator-based method requires an electron beam energy of at least $150 \mathrm{GeV}$ for full design positron beam intensity. Therefore at centerof-mass energies below $300 \mathrm{GeV}$ the luminosity is reduced due to a lower positron beam current.

Both beams are injected into damping ring at $5 \mathrm{GeV}$ energy. The bunch train is stored in the ring in a compressed mode, with the bunch spacing reduced by a factor 17; even with this compression, a large ring circumference of about $17 \mathrm{~km}$ is needed. To avoid building an additional large ring tunnel, a so-called "dog bone" geometry is used (see Fig.8). The damping ring optics is designed to achieve $8 \cdot 10^{-6} \mathrm{~m}$ horizontal and $0.02 \cdot 10^{-6} \mathrm{~m}$ vertical emittances (normalized), providing a 50\% margin for vertical emittance degradation until the IP. About $400 \mathrm{~m}$ of wiggler section are needed to achieve sufficient damping time ( $28 \mathrm{~ns})$. Fast kickers are required for compression and decompression of the bunch train at injection and extraction respectively.

Despite its unconventional shape, the damping ring does not exhibit any unusual beam dynamics. The only exception, related to its large circumference, is a large incoherent space charge tune shift. The effect can be significantly reduced, however, by raising the beam energy and artificially increasing the beam cross-section in the long straight sections which dominates the space charge effect. To do so, the beam is transversely fully coupled in the straight sections by inserting skew quadrupoles. 


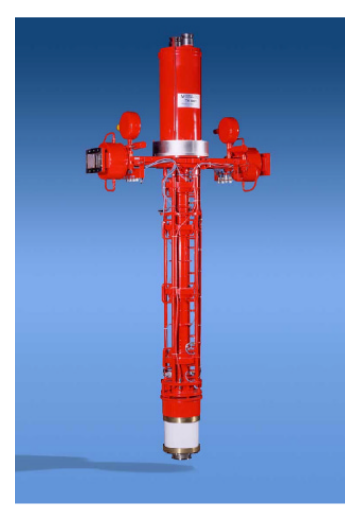

Figure 11: The Thomson TH1801 multibeam klystron.

\subsection{Main Linac}

The two main linear accelerators are each constructed from roughly ten-thousand one-meter long superconducting cavities. Groups of twelve cavities are installed in a common cryostat (cryomodule) whose design is based on that successfully used in the TTF, modified to be more modular and cost-effective. As shown by Fig.10, groups of 3 cryomodules, or 36 cavities, are powered by a single $10 \mathrm{MW}$ multibeam klystron with two RF output windows. Such a klystron (see Fig.11) with seven beams has been tested at $10 \mathrm{MW}$ with an efficiency close to $70 \%$. In the baseline design, the coaxial coupler must transmit about $230 \mathrm{~kW}(24 \mathrm{MV} \times 9.5 \mathrm{~mA})$ peak RF power, well below the 1.6 MW achieved by TTF couplers. The cryogenic system for the TESLA linac is comparable in size and complexity to the one currently under construction for the LHC at CERN.

Beam dynamics studies have specified the realistic linac tolerances on the cavity and quadrupole alignment after installation and survey of the linac modules: $0.5 \mathrm{~mm}$ (rms) for the cavities and $0.3 \mathrm{~mm}(\mathrm{rms})$ for the quadrupoles. As shown by Fig.12, the single bunch emittance growth resulting from short range wakefields excited in misaligned cavities is about $7 \%$, with an additional $6 \%$ for a one $\sigma_{y}$

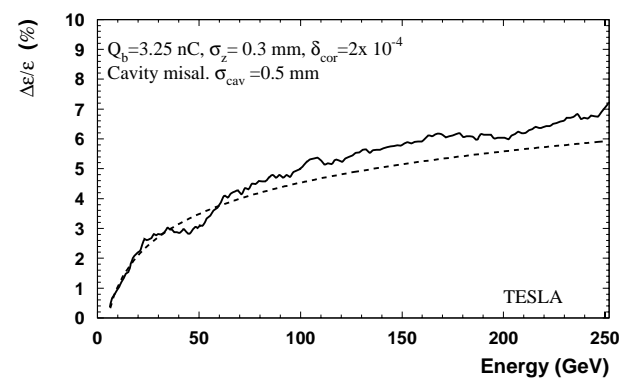

Figure 12: Vertical emittance growth obtained from simulation of 50 random cavity misalignments $\left(\delta y_{c}=0.5 \mathrm{~mm}\right.$ rms). The dashed curve is the analytical prediction from a two-particle model.

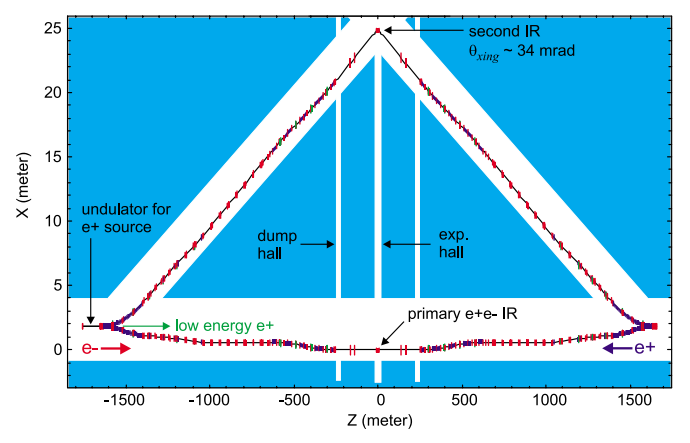

Figure 13: Geometry of the BDS, including the second IR.

injection error. The dispersive effect of quadrupole misalignments can be much larger unless it is compensated by beam-based alignment methods. Relying on a BPM resolution of $10 \mu \mathrm{m}$ (rms), beam-based correction algorithms already used at the SLC limit the dispersive emittance growth to a few $\%$. These effects are well contained within the $50 \%$ margin in vertical emittance coming from the damping rings.

With a train of bunches, the long-range wakefields excited by each bunch will act on the subsequent bunches, resulting in a possible multi-bunch emittance growth. In order to reduce the effective wake along the TESLA bunch train, a small damping of the higher order modes (HOM) is required. The lifetime of the modes is reduced by means of HOM couplers which are mounted at both ends of a TESLA cavity. The multi-bunch transverse distribution resulting from a given set of cavity alignment errors is significant only in the head of the bunch train and the emittance growth integrated over the complete bunch train is negligible. Moreover, its pattern is stable with time which opens the possibility to remove most of it by a fast intra-pulse orbit correction system.

\subsection{Beam Delivery System}

The complete beam delivery system (BDS) layout (linac to linac) is shown in Fig.13, including the optional second IP. It consists of beam switch-yard, collimation, beam diagnostics and correction and final focus sections. By putting the undulator based $e^{+}$source in front of the electron beam switch-yard, both IP's can host $e^{+} e^{-}$collisions, while $\gamma \gamma$ collisions which require a non zero crossing angle can take place only at the second IP. The main extraction beam line transports the spent beams almost loss-free to the dump halls, on both sides of the IP. An fast emergency extraction line, starting in front of the collimation section, brings the beams to the main dump system. It is primarily intended to extract some fraction of the bunch train in the event of a machine protection trip. It will also serve as a by-pass line during commissioning.

Due to the high vertical disruption parameter at the IP ( $D_{y} \approx 25$ ), the luminosity is extremely sensitive to small offsets in both beam-beam displacement and crossing angle. As a result, the collisions must be maintained to 


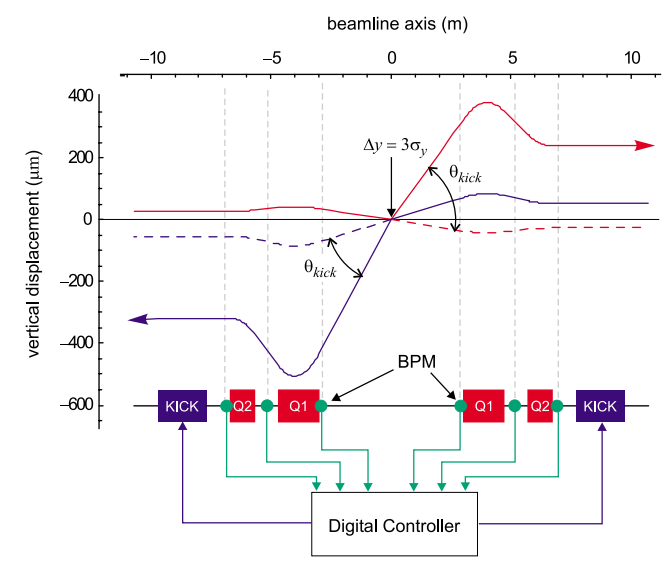

Figure 14: The IP fast feedback system. The red and blue rays represent an example having a $3 \sigma_{y}^{*}$ offset at the IP (corresponding approximately to a $10 \sigma_{y^{\prime}}^{*}$ kick).

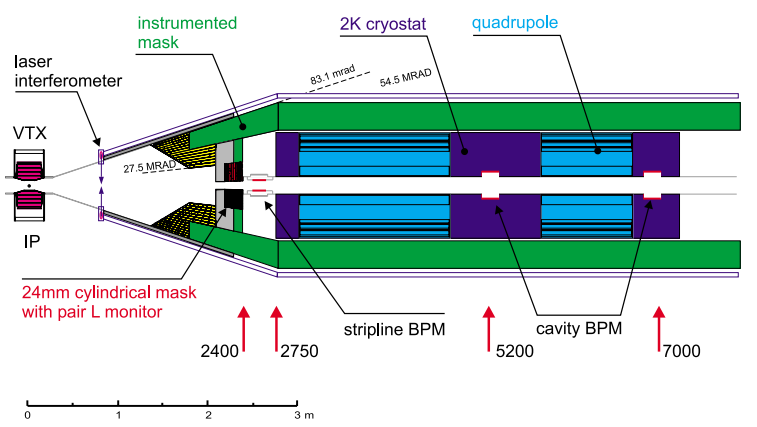

Figure 15: Interaction region layout.

within $0.1 \sigma$ in both offset and angle $(0.5 \mathrm{~nm}$ and $1.2 \mu \mathrm{rad}$ respectively). Figure 14 illustrates the concept for the IP beam separation feedback system. The large TESLA bunch spacing of 337 ns allows the use of a digital controller. Fast kickers $(\sim 100 \mathrm{~ns})$ are used to make the necessary orbit corrections. The feedback signal is derived directly from the strong beam-beam kick which both beams experience when they do not collide head-on. Such a feedback system, successfully used at the SLC[5], can correct up to $200 \mathrm{~nm}$ motion of the last doublet at frequencies $f \ll 3 \mathrm{MHz}$ with a luminosity loss smaller than $10 \%$. The implementation of the high resolution BPM's ( $5 \mu \mathrm{m}$ on a bunch to bunch basis) within the final doublet and IP region is shown in Fig.15. The tungsten mask shown in this figure shields the detector from the copious $e^{+} e^{-}$pair background produced in the beam-beam interaction. It also supports a fast luminosity monitor using the incoherent $e^{+} e^{-}$pair signal. This detector, able to accurately measure bunch to bunch luminosity variations, provides a powerful tool to optimize the collider and can be included in the fast feedback algorithm.

\section{COST AND TIME SCHEDULE}

The cost of the $500 \mathrm{GeV}$ linear collider with one interaction point is summarized in Table 2. Adding
Table 2: TESLA collider costs in M Euro (one IP).

\begin{tabular}{lc}
\hline Main LINAC Modules & 1,131 \\
Main LINAC RF System & 587 \\
Civil Engineering & 546 \\
Machine Infrastructures & 336 \\
Damping Rings & 215 \\
Auxiliary Systems & 124 \\
HEP Beam Delivery & 101 \\
Injection System & 97 \\
\hline Collider Total & 3,136 \\
\hline
\end{tabular}

$531 \mathrm{M}$ Euro for X-FEL accelerator increment and laboratory and $210 \mathrm{M}$ Euro for the high energy physics detector, the total cost of the TESLA project is 3,877 M Euro, with no contingency. The cost of the accelerating modules complies with the $2 \mathrm{k} \$ / \mathrm{MV}$ target set by B. Wiik in 1992 . It is based on TTF experience and obtained from industrial studies made by companies with expertise in $\mathrm{Nb}$ cavity fabrication and mass production plants.

The construction time is foreseen to be 8 years split roughly into 4 years of tunneling (with 4 boring machines in parallel) and civil construction, and 4 years of installation, with $30 \mathrm{~m}$ of accelerator structure installed per day in the last 3 years. The manpower required during construction time is expected to culminate to about 1,000 FTE/yr in the last 4 years of construction. The cost of operating 5000 hours/year is estimated to $120 \mathrm{M}$ Euro/year.

\section{CONCLUSION}

Convinced by the reliability and the potential of the TESLA technology, the TESLA collaboration released a Technical Design Report (TDR) in March 2001. This report includes a cost estimate for the collider, as well as an implantation study on the DESY site. The TDR started the process of project evaluation at a national (submission to the German Science Council in 2001) and international level before an international collaboration can be formed.

\section{REFERENCES}

[1] R. Brinkmann, K. Flöttmann, J. Roßbach, P. Schmüser, N. Walker and H. Weise (eds.), "TESLA Technical Design Report, Part II The Accelerator”, DESY 2001-011, http://tesla.desy.de/new_pages/TDR_CD/PartII/accel.html

[2] "Proposal for a TESLA Test Facility”, DESY-TESLA-93-01, 1992.

[3] M. Hüning, "Recent Results from the Tesla Test Facility (TTF)", WOPA012 this conference.

[4] R. Alley, et.al., "The Stanford Linear Accelerator Polarised Electron Source”, Nucl. Inst. and Meth. A 365, p.1, 1995.

[5] F. Rouse, T. Gromme, W. Kozanecki, N. Phinney, "Maintaining Micron Size Beams in Collision at the Interaction Point of the Stanford Linear Collider", SLAC-PUB-5512 (1991) 